\title{
Midinfrared Conductivity in Orientationally Disordered Doped Fullerides
}

\author{
M. S. Deshpande and E. J. Mele \\ Department of Physics \\ Laboratory for Research on the Structure of Matter \\ University of Pennsylvania, Philadelphia, PA 19104 \\ M. J. Rice \\ Xerox Webster Research Center \\ Webster, New York 14580 \\ H-Y Choi \\ Department of Physics \\ Sung Kyun Kwan University \\ Suwon 440746 Korea
}

\begin{abstract}
The coupling between the intramolecular vibrational modes and the doped conduction electrons in $M_{3} C_{60}$ is studied by a calculation of the electronic contributions to the phonon self energies. The calculations are carried out for an orientationally ordered reference solid with symmetry $F m \overline{3} m$ and for a model with quenched orientational disorder on the fullerene sites. In both cases, the dispersion and symmetry of the renormalized modes is governed by the electronic contributions. The current current correlation functions and frequency dependent conductivity through the midinfrared are calculated for both models. In the disordered structures, the renormalized modes derived
\end{abstract}


from even parity intramolecular phonons are resonant with the dipole excited single particle spectrum, and modulate the predicted midinfrared conductivity. The spectra for this coupled system are calculated for several recently proposed microscopic models for the electron phonon coupling, and a comparison is made with recent experimental data which demonstrate this effect.

PACS Numbers : 78.20.-e, 74.70 Ya, 74.20.-z

\section{INTRODUCTION}

Soon after the discovery of solid phases of $C_{60}$ [1] it was demonstrated that these solids can be doped from an insulating parent phase through a series of interesting conducting and nonconducting phases by the introduction of alkali species in the interstitial volume [2]. There has been particular interest in the properties of the family of $M_{3} C_{60}$ (where $\mathrm{M}$ is an alkali metal) which exhibit superconducting ground states. The interplay of the superconductivity and the unique structural properties exhibited by these molecular metals is a key issue for these materials.

Among the various proposed theories for the superconductivity of these phases is a model which posits that an effective attractive interaction between the conduction electrons is mediated by exchange of intramolecular phonons in the doped solid [3, 4]. Since this model was

originally proposed, it has been appreciated that the theory can be tested quantitatively by examining the renormalization of the intramolecular vibrational modes which derive from their coupling to the doped conduction electrons. Indeed, the key Raman active vibrational modes are experimentally found to shift and broaden in the conducting state [5] [7], confirming the underlying idea that coupling between the intramolecular modes and the conduction charge is important for the physics of the doped phases.

This theoretical picture is complicated in an essential way by the orientational "merohe- 
dral" disorder [8] of the alkali doped samples which have been synthesized. Experimentally, the structure of most of the $M_{3} C_{60}$ samples appears to be that of an orientational glass. In these solids, each of the $C_{60}$ molecules, on the lattice sites of an FCC Bravais lattice, adopts one of tho high symmetry orientations with the two fold molecular axes oriented along the (001) crystal directions. There are two inequivalent ways in which each molecule can achieve this orientation, and they are related by a $\pi / 2$ rotation about any (001) direction. This choice of orientation varies, essentially randomly, from site to site in the solid.

This structural disorder has important consequences for virtually all the electronic properties of the doped solid, including the renormalization of the intramolecular modes mentioned above. Nevertheless, the effects of the disorder are awkward to treat properly in the theory, and consequently most theoretical work has treated the effects of orientational disorder only very approximately. Indeed, a considerable body of work has investigated the "zero band width" limit of this problem [3, [4, 9], in which the intermolecular hopping amplitudes are set to zero and progress can be made analytically. This approximation is based on the fact that the intermolecular electronic hopping amplitudes are much smaller than the intramolecular amplitudes which allows a useful separation of energy scales. Thus many solid state properties do reflect the underlying molecular structure of the solid. For example, the conduction band relevant to our discussion below is derived primarily from the antibonding $t_{1 u}$ molecular state [10], with very little mixing between different intramolecular orbitals in the solid phase. Similarly, each of the vibrational bands in the solid may be identified with a fundamental vibrational excitation of the isolated $C_{60}$. This intramolecular symmetry also strongly constrains the possible couplings between the vibrational degrees of freedom and the conduction electrons : electronic density fluctuations on a given fullerene site may be coupled only to the fully symmetric $a_{g}$ or quadrupolar $h_{g}$ vibrational excitations on the same site [3].

Of course the zero band width limit does not address any phenomenon which derives from the intermolecular properties of the doped solid. For example, the two point density density response function for the doped carriers depends explicitly on the three dimensional 
dynamics of the doped carriers in these molecular solids. This in turn depends sensitively on the degree and type of microscopic structural disorder in the doped phases, so that spectroscopy on the low energy electronic excitations can be used to probe the structural disorder in the doped solids [11,12]. In this theory, scattering from static orientational fluctuations in the doped solid leads to a residual mean free path for the conduction charge of order $20 \mathrm{~A}$ [13], and to a residual resistivity of order $3000 \mathrm{~S} / \mathrm{cm}$ [12]. This strong scattering is believed to dominate the observed low frequency conductivity which is still lower, of order $1500 \mathrm{~S} / \mathrm{cm}$ [14,15] at low temperatures.

The phonon renormalizations mentioned previously are obtained from a similar family of two point functions, and are also quite sensitive to the intersite orientational correlations of the solid. For a reference orientationally ordered solid, the high symmetry of the structure leads to selection rules which are applicable to the solid phase and which further restrict the coupling of the vibrational and electronic degrees of freedom. These selection rules can be violated in the disordered solid, depending on the type and degree of the orientational disorder. This is very important for understanding the renormalization of the intramolecular vibrations in both the ordered and disordered phases, and the response functions derived from these modes [16]. In fact, the spectrososcopic signatures of this effect are now beginning to be reported in experiments for the doped fullerides where several of the even parity "on ball" vibrations can be observed as modulations in the dipole active long wavelength and low frequency electronic excitation spectra. This is strictly forbidden for the orientationally ordered structure, and its appearance in experiments provides a powerful direct probe of the scattering by oreintational fluctuations in the doped phases. This effect is reminscent of similar phenomena which have been previously encountered in the charge transfer salts [17 19], and in the doped conducting polymers [20], although the structural origins of the symmetry breaking here are somewhat unique to the fullerides.

In this paper, we present a study of the electron phonon coupling in the doped solids in which we explicitly treat the three dimensional dynamics of the doped conduction charge. This theory is carried out first for a reference orientationally ordered structure, and then for 
a model with orientational randomness. Below we calculate the electronic contributions to the phonon self energies in both models, so that the effects on orientational disorder on the phonon self energies can be clearly identified. Finally, we compute the phonon contributions to the long wavelength current current response function, and study the infrared conductivity

for this coupled electron phonon system. A brief comparison with experiments is given at the end of the paper. There we find that the mid-infrared conductivity for the coupled system depends sensitively on the disorder and the distribution of electron phonon coupling strengths, and may therefore be used to critically compare several popular models for the electron phonon coupling constants for these materials.

\section{DISORDER AND ELECTRONIC STATES IN $M_{3} C_{60}$}

In this section we review the tight binding description of conduction electronic states in the doped fullerides. Since this construction has been presented in detail elsewhere [11,21], our presentation will be brief.

The conduction band of the alkali doped fullerides is derived from an antibonding molecular orbital of the isolated $C_{60}$ with $t_{1 u}$ symmetry. This is the LUMO of the neutral molecule; it is three fold degenerate, and transforms like a vector under spatial rotations. The band structure of the doped and undoped orientationally ordered phases has been studied by first principles methods 222 24]. For both cases it is found that the conduction bands can be described reasonably well by a model restricted to hopping of a three component field $\Psi_{i}=$ $\left(c_{i \mu}, \mu=x, y, z\right)$ between nearest neighbor fullerene sites:

$$
H_{e l}=\sum_{i, j} \sum_{\mu \nu} T_{\mu \nu}(i j) c_{i \mu}^{\dagger} c_{j \nu}
$$

where $c_{i \mu}^{\dagger}$ creates an electron at site $i$ in the $\mu$ th orbital. The matrix elements in $\mathrm{T}$ depend on the orientation of the bond between sites $i$ and $j$, on the orbital polarizations of the two sites connected in the hop, and on the molecular orientations of the terminal sites. In accordance with experiment [8] we will restrict our attention to the situation where the terminal sites 
can adopt either of the two high symmetry settings with the two fold axes aligned along the (100) crystal directions, which we label the "A" and "B" orientations. The choice of setting can be indexed by an Ising spin variable, $s_{i}$ defined on each site and which takes the value $s_{i}=1(-1)$ for the $\mathrm{A}(\mathrm{B})$ orientation. The intermolecular hopping amplitude then depends on the spin variables on neighboring sites in the manner,

$$
\begin{aligned}
T(i j)= & T^{(0)}\left(\tau_{i j}\right)+T^{(1)}\left(\tau_{i j}\right) s_{i}+T^{(2)}\left(\tau_{i j}\right) s_{j} \\
& +T^{(3)}\left(\tau_{i j}\right) s_{i} s_{j}
\end{aligned}
$$

So that the hopping amplitude can be developed from the four fundamental hopping matrices $T^{(n)}$. For a hop along a reference bond $\overrightarrow{\tau_{i j}}=(a / 2)(\hat{i}+\hat{j}) \equiv \overrightarrow{\tau_{o}}$ the matrix $\mathrm{T}$ take the form

$$
\begin{gathered}
T^{(0)}\left(\overrightarrow{\tau_{o}}\right)=t\left[\begin{array}{ccc}
A & B & 0 \\
B & A & 0 \\
0 & 0 & C
\end{array}\right] T^{(1)}\left(\overrightarrow{\tau_{o}}\right)=t\left[\begin{array}{ccc}
X & Y & 0 \\
-Y & -X & 0 \\
0 & 0 & 0
\end{array}\right] \\
T^{(3)}\left(\overrightarrow{\tau_{o}}\right)=t\left[\begin{array}{ccc}
X & -Y & 0 \\
Y & -X & 0 \\
0 & 0 & 0
\end{array}\right] T^{(4)}\left(\overrightarrow{\tau_{o}}\right)=t\left[\begin{array}{ccc}
D & E & 0 \\
E & D & 0 \\
0 & 0 & F
\end{array}\right]
\end{gathered}
$$

where the three basis orbitals are assigned to the $\mathrm{x}, \mathrm{y}$, and $\mathrm{z}$ orbital polarizations, respectively. The hopping amplitudes for any other bond direction can be obtained by a rotation of the T's listed in equation 3. The values of the parameters A, B, C, D, E and F, deduced from the work of Gelfand and $\mathrm{Lu}$, are listed in table 1. We should note that our convention for assigning the orbital polarizations is fixed in the crystal frame and therefore does not change when the molecular orientation is rotated. This is in fact different from the convention originally adopted by Gelfand and $\mathrm{Lu}$, and the reader is directed to the Appendix A of Ref. 21] for a discussion of this point.

Below we will investigate the effects of orientational disorder in the supercell approximation in which we construct a periodically repeated unit cell containing a large (typically 32) molecules with the orientations randomly assigned to the A and B settings. The electronic 
Hamiltonian for this model is developed from the fundamental hopping matrices listed in equation 3. The artificial periodic boundary conditions allow us to analyze this problem in the Bloch orbital basis :

$$
c_{k, i \mu}^{\dagger}=\frac{1}{\sqrt{N}} \sum_{I} e^{i k \cdot R_{I}} c_{I, i \mu}^{\dagger}
$$

in a solid with $\mathrm{N}$ supercells, indexed by the supercell translation vectors $R_{I}$, and where $\mathrm{i}$ and $\mu$ index the basis site and orbital polarization respectively. In this basis the Hamiltonian for a supercell containing $\mathrm{M}$ sites is a $3 \mathrm{M}$ x $3 \mathrm{M}$ matrix $\mathrm{H}(\mathrm{k})$, for which the eigenvectors $C_{n k}^{\dagger}$ are expanded in the Bloch orbital basis:

$$
C_{n k}^{\dagger}=\sum_{i \mu} A_{n, i \mu}(k) c_{k, i \mu}^{\dagger}
$$

Several authors [11 13] have applied this model to study the conduction electron states in orientationally ordered and disordered structural models. The fluctuations in intersite terms in $H_{e l}$ due to the disorder in this problem are on the order of the Fermi energy, and so one can correctly anticipate that the effects of disorder will be quite significant. Explicit calculations demonstrate that the full conduction bandwidth in the disordered phase is only slighly smaller than the bandwidth obtained for the orientationally ordered structure and in addition the density of states at the Fermi energy is not significantly different from the value for the ordered structure. However, the electrons are strongly scattered by the orientational fluctuations, and the calculated conduction band spectrum for the disordered alloy is smooth and relatively structureless. A closer inspection of the underlying conduction electron states in the disordered orientational alloy reveals that electron propagation near the Fermi level can be well described in terms of an effective Bloch wavevector $\mathrm{k}$, and a mean free path of order $20 \mathrm{~A}$ [13]. There is no evidence in the theory for localization of the one electron states in this model except in a narrow range of energy near the band extrema which is irrelvant for electronic processes near the Fermi energy. This is in accord with experimental measurements which show a metallic conductivity at low temperature in the normal phase [15], that is the measured or extrapolated conductivity at low temperature comfortably 
exceeds the minimum metallic conductivities expected at these electron densities. Thus theory and experiment provide a consistent overall picture that the electronic structure near the Fermi energy for the disordered alloy is that of a "dirty" metal with propagating states at the Fermi energy and a relatively short residual mean free path.

\section{INTRAMOLECULAR PHONONS}

In this section we present the model for the "bare" unrenormalized phonons of the doped solid. The 176 modes of a single fullerene molecule can be indexed using the ten irreducible representations of the icosahedral group $I_{h}$ [25,26]. However, the conduction states are derived from the $t_{1 u}$ molecular states, and therefore the conduction charge in this manifold may couple only to the lattice modes of $a_{g}$ and $h_{g}$ symmetry. There are two such fully symmetric modes, they are the molecular breathing mode and pentagonal pinching mode of the icosahedral molecule. There are eight families of $h_{g}$ modes, and these describe the various possible quadrupolar deformations of the $C_{60}$ molecule. These intramolecular modes span an energy range from $200 \mathrm{~cm}^{-1}$ to $1600 \mathrm{~cm}^{-1}$.

In our model, the bare phonon Hamiltonian will be represented by the Einstein spectrum:

$$
H_{p h}=\sum_{i} \sum_{\alpha m} \omega_{\alpha m}\left(b_{\alpha m}^{\dagger}(i) b_{\alpha m}(i)+\frac{1}{2}\right)
$$

where $b_{\alpha m}^{\dagger}(i)$ creates the m-th mode of symmetry $\alpha$ on the i-th site. We shall use a convention where $\alpha=0($ with $m=1,2)$ denotes the $a_{g}$ modes and $\alpha=1,5($ with $m=1,8$ ) label the $h_{g}$ quintuplets. The degeneracy of each $h_{g}$ quintuplet implies that for $\alpha=1,5$, $\omega_{\alpha m}=\omega_{m}$. These even parity modes are experimentally accessible by Raman scattering, and measurements of the Raman frequencies for $M_{x} C_{60}$ with $\mathrm{x}=0,3$ and 6 have now been reported from several groups [5 [7]. The striking result of the experiments is that the Raman lines in $M_{3} C_{60}$ are shifted and broadened relative to the undoped solid. On the other hand in $M_{6} C_{60}$, which is an insulator, although the frequencies are further shifted, the Raman lines are again sharp. It is suggested that the source of the shift and broadening in the $M_{3} C_{60}$ 
phase is primarily the coupling of the intramolecular modes of equation 6 to the conduction charge. For the $M_{6} C_{60}$ structure which is a band insulator, the charge gap is of order 0.5 $\mathrm{eV}$, so that the phonon self energy is again purely real, leading to a shift of the frequency but no damping.

There are several important contributions to the frequency shifts and linewidths of the Raman active vibrations of the doped system. Most notably, the intramolecular interatomic potential is intrinsically anharmonic. Thus addition of charge, even to an isolated molecule, will lead to a relaxation of the bond lengths, and to a shift of the bare frequency. Here "bare" implies that the phenomenon is not a solid state, but rather a molecular effect. Note that this effect is not accompanied by electronic induced broadening which is a solid state effect. The frequency renormalization in the $M_{6} C_{60}$ insulating phase is due to just such an "intramolecular" effect.

The situation in the interesting $M_{3} C_{60}$ phases is complicated due to the confluence of these "intramolecular" and "intermolecular" solid state processes. The added conduction charge induces an intramolecular relaxation of the bond lengths and frequency shifts of the type observed for the $M_{6} C_{60}$ phases [29,28,27]. There are, in addition, self energy contributions due to the "one loop" density fluctuations of the added conduction electrons. (These are clearly abent for the terminal phase with $\mathrm{x}=0$ which has no conduction charge, and for the $\mathrm{x}=6$ phase which has a saturated band). These terms are nevetheless the most interesting since they communicate information about the solid state structure, and particularly about the intersite orientational correlations, into our effective Hamiltonian for the lattice degrees of freedom. These terms are clearly essential for what follows as they induce a mixing, frequency shift and damping of the coupled intramolecular modes.

For simplicity we shall assume that the two types of phonon renormalizations, intermolecular (due to the one loop corrections from the conduction charge) and intramolecular (due to the anharmonic terms in the bare Hamiltonian and to higer order terms in the electron phonon coupling) are simply additive. Thus the bare Hamiltonian of equation 6 will be assumed to already contain the intramolecular effects, and we will explicitly calculate the 
intermolecular contributions absent from that model. To be precise, Faulhaber et. al. 28] have reported theoretical results for the lattice vibrations of the isolated $C_{60}^{3-}$ trianion, which properly contain the intramolecular contributions only, and we adopt their results for the frequencies $\omega_{\alpha n}$ appearing in equation 6. For convenience, these frequencies are reproduced in Table 2. We then proceed to explicitly evaluate the important "solid state" one loop electronic contributions to our effective Hamiltonian in the doped phases.

\section{ELECTRON PHONON INTERACTION}

The lattice modes of equation 6 are coupled linearly to the conduction electrons via an on site deformation potential [3,9]:

$$
H_{e l-p h}=\sum_{i} \sum_{\lambda} \sum_{\mu \nu} \Gamma_{\mu \nu}^{\lambda} c_{\mu i}^{\dagger} c_{\nu i} Q_{\lambda}(i)
$$

where

$$
Q_{\lambda}(i)=b_{\lambda}(i)+b_{\lambda}^{\dagger}(i)
$$

is the displacement corresponding to the $\lambda-t h$ mode on site i. Here $\Gamma_{\mu \nu}^{\lambda}$ is a matrix acting in the electronic $t_{1 u}$ subspace. The symmetry of this matrix is fixed solely by the symmetry of the coupled mode $\lambda$ while the strength of the interaction depends on the particular mode of interest. If we write $\lambda=\alpha m$ for the m-th mode of symmetry type $\alpha$ we have that

$$
\Gamma_{\mu \nu}^{\alpha n}=g_{\alpha m} \Delta_{\mu \nu}^{\alpha}
$$

where $\alpha=0$ denotes a fully symmetric $a_{g}$ mode and $\alpha=(1, . ., 5)$ denote the five basis functions in the $h_{g}$ representation of $I_{h}$. Anticipating our passage to a crystal with cubic symmetry we will break this manifold into the invariant $e_{g}$ and $t_{2 g}$ subspaces and write:

$$
a_{g}: \quad \quad \Delta^{0}=\left(\begin{array}{ccc}
\frac{1}{\sqrt{3}} & 0 & 0 \\
0 & \frac{1}{\sqrt{3}} & 0 \\
0 & 0 & \frac{1}{\sqrt{3}}
\end{array}\right)
$$




$$
\begin{gathered}
t_{2 g}: \Delta^{1}=\left(\begin{array}{ccc}
0 & \frac{1}{\sqrt{2}} & 0 \\
\frac{1}{\sqrt{2}} & 0 & 0 \\
0 & 0 & 0
\end{array}\right) \Delta^{2}=\left(\begin{array}{ccc}
0 & 0 & 0 \\
0 & 0 & \frac{1}{\sqrt{2}} \\
0 & \frac{1}{\sqrt{2}} & 0
\end{array}\right) \\
\Delta^{3}=\left(\begin{array}{ccc}
0 & 0 & \frac{1}{\sqrt{2}} \\
0 & 0 & 0 \\
\frac{1}{\sqrt{2}} & 0 & 0
\end{array}\right) \\
e_{g}: \Delta^{4}=\left(\begin{array}{ccc}
\frac{1}{\sqrt{6}} & 0 & 0 \\
0 & \frac{1}{\sqrt{6}} & 0 \\
0 & 0 & -\frac{2}{\sqrt{6}}
\end{array}\right) \Delta^{5}=\left(\begin{array}{ccc}
\frac{1}{\sqrt{2}} & 0 & 0 \\
0 & -\frac{1}{\sqrt{2}} & 0 \\
0 & 0 & 0
\end{array}\right)
\end{gathered}
$$

We have defined these matrices so that they are orthonormal with $\operatorname{tr} \Delta^{\alpha} \Delta^{\beta}=\delta_{\alpha \beta}$. As expected, the form of the $\Delta$ matrices requires that the symmetric modes couple linearly to the total on ball conduction charge density and the $h_{g}$ modes are linearly coupled to the electric quadrupole density fluctuations.

The $g_{\alpha m}$ 's appearing in equation 9 are the deformation potentials are obtained by studying the linear variation of the intramolecular Hamiltonian with respect to the normal coordinates of the vibrational mode of interest.

$$
g_{\alpha m} \Delta_{\mu \nu}^{\alpha}=\frac{\hbar}{M \omega_{\alpha m}}\left\langle\mu\left|\frac{\partial H_{m o l}}{\partial Q_{\alpha m}}\right| \nu\right\rangle
$$

where $H_{m o l}$ is the intramolecular electronic Hamiltonian and $\mathrm{M}$ is the mass of a carbon atom. A microscopic evaluation of these coupling coefficients requires a model for the dependence of $H_{m o l}$ on intramolecular displacements, as well as a microscopic assignment of the various intramolecular phonon frequencies and eigenvectors. There have been several evaluations of the $g_{\alpha n}$ 's reported in the literature [3,4,28] which treat $H_{m o l}$ and the bare vibrational modes at various levels of sophistication. The coefficients $g_{\alpha n}$ reported in these studies are displayed in table 3. Note that for $h_{g}$ modes $(\alpha=1,5), g_{\alpha m}=g_{m}$. We also note that the data tabulated here differ from those reported in several of the original papers owing to different normalization conditions on the $\Delta$ matrices adopted by the various authors. These 
various models may be classified according to two groups: those that localize the strongest interactions to the two highest frequency $h_{g}$ modes and those that distribute the coupling more uniformly over the phonon spectrum. The two highest frequency $h_{g}$ modes contain double bond stretching character, and therefore might be expected to couple strongly to the added conduction charge which occupies an antibonding state. The coupling to the lower frequency modes is a more subtle matter, deriving from the perturbation to $H_{m o l}$ due to bond angle fluctuations.

The expression for $H_{e l-p h}$ is valid for an isolated molecule. Information about the solid state properties is then contained in the electronic amplitudes $\left\langle c_{\mu i}^{\dagger} c_{\nu i}\right\rangle$ appearing in this expression. Using the notation in Section II for a periodically repeated supercell, the linearized interaction reads

$$
H_{e l-p h}=\sum_{I} \sum_{\lambda i} \sum_{\mu \nu} \Gamma_{\mu \nu}^{\lambda} c_{I i \mu}^{\dagger} c_{I i \nu} Q_{\lambda i}(I)
$$

Transforming to the basis of Bloch eigenstates $C_{n k}^{\dagger}$ given by the expression,

$$
C_{n k}^{\dagger}=\sum_{I i \mu} A_{n, i \mu}(k) e^{i k \cdot R_{I}} c_{I, i \mu}^{\dagger}
$$

we obtain,

$$
H_{e l-p h}=\sum_{k, n, k^{\prime}, n^{\prime}} \sum_{\lambda i} \tilde{\Gamma}_{n n^{\prime}}^{\lambda i}\left(k, k^{\prime}\right) C_{n i}^{\dagger}(k) C_{n^{\prime} i}\left(k^{\prime}\right) Q_{\lambda i}\left(k-k^{\prime}\right)
$$

where,

$$
Q_{\lambda i}(q)=b_{\lambda i}(-q)+b_{\lambda i}^{\dagger}(q)
$$

and ,

$$
\tilde{\Gamma}_{n, n^{\prime}}^{\lambda i}\left(k, k^{\prime}\right)=\sum_{\mu \nu} \Gamma_{\mu \nu}^{\lambda} A_{n i \mu}^{\star}\left(k^{\prime}\right) A_{n^{\prime} i \nu}(k)
$$

is the electron-phonon coupling evaluated in the Bloch basis. The phonons relevant to our discussion of the infrared absorption spectra are those with $\mathrm{q}=0$ and hence we will set $\mathrm{k}=$ $\mathrm{k}^{\prime}$ in the above expressions and abbreviate the diagonal components $\tilde{\Gamma}_{n, n^{\prime}}^{\lambda i}(k, k)$ by $\tilde{\Gamma}_{n, n^{\prime}}^{\lambda i}(k)$ and $Q_{\lambda i}(0)$ by $Q_{\lambda i}$. 


\section{PHONON SELF ENERGIES}

In this section we calculate the one loop corrections to the dynamical matrix for the phonons in the $\mathrm{x}=3$ phase. The calculations are carried out first for a reference orientationally ordered model, and then for a model containing quenched disorder. We focus on the selection rules governing the electron phonon coupling for the ordered case, and the breakdown of these rules in the orientationally disordered phase. We also compute the renormalized phonon Green's function which includes the effects of the one loop corrections and which will be used to calculate the current current correlation function for the coupled electron phonon system in Section VI(b).

We will study the phonon Green's function

$$
D\left(\lambda i, \lambda^{\prime} i^{\prime} ; \omega\right)=-i \int_{0}^{\infty} d t e^{-i \omega t}\left\langle T\left[Q_{\lambda i}, Q_{\lambda^{\prime} i^{\prime}}^{\dagger}\right]\right\rangle
$$

As in the previous section, $\lambda$ indexes the a particular symmetrized on ball displacement field, and i indexes a site in the unit cell. We specialize the discussion to the $q=0$ part of the Green's function, and therefore enforce periodic boundary conditions on the displacement

field $Q_{\lambda i}^{\dagger}+Q_{\lambda i}$. In the absence of the electron phonon interaction the unrenormalized Green's function is diagonal in both the site and phonon "band" indices $\lambda$.

$$
D_{o}\left(\lambda i, \lambda i^{\prime} ; \omega\right)=D_{o}(\lambda, \omega) \delta_{\lambda \lambda^{\prime}} \delta_{i i^{\prime}}
$$

where

$$
D_{o}(\lambda, \omega)=\frac{2 \omega_{\lambda}}{\omega_{\lambda}^{2}-\left(\omega-i \epsilon_{\lambda}\right)^{2}}
$$

This Green's function is assumed to contain all the relevant intramolecular renormalizations, which are then parameterized by the bare frequencies $\omega_{\lambda}$ and the linewidths $\epsilon_{\lambda}$. For the former we use the theoretical data of Ref. [28], while for the latter we use the experimentally observed linewidths for undoped $C_{60}$ reported in [7]. These parameters are listed in Table 3. 
The linear electron phonon interaction in equation 0 is represented by the elementary vertex shown in Figure 1(a). In the Bloch orbital basis, this matrix element is diagonal in the site index but in general not in the orbital indices $\mu$. This scattering amplitude provides a second order contribution to the phonon self energy, where the relevant term describes a process whereby an electron hole pair, created on a common site $i$ by the vertex shown in Figure 1(a) propagate and ultimately recombine on a site $i^{\prime}$ in the solid. The vibrational degrees of freedom on different sites are therefore coupled indirectly through the density fluctuations of the conduction electrons. This process, represented diagramatically in Figure 1(b), is evaluated to give the self energy contribution

$$
\begin{aligned}
& \Pi\left(\lambda i, \lambda^{\prime} i^{\prime} ; \omega\right)=\sum_{k} \sum_{n \neq n^{\prime}} \frac{\tilde{\Gamma}_{n n^{\prime}}^{\lambda i}(k) \tilde{\Gamma}_{n^{\prime} n}^{\lambda^{\prime} i^{\prime}}(k)}{E_{n k}-E_{n^{\prime} k}-\omega-i \delta} \\
& \left(f\left(E_{n k}\right)-f\left(E_{n^{\prime} k}\right)\right)
\end{aligned}
$$

For this molecular solid in which the "bare" phonons are well described by Einstein oscillators with negligible direct intersite coupling the indirect coupling in equation 20 determines the dispersion of the vibrational bands of the doped phase. Similarly, all the information about the orientational structure of the solid phase enters our theory through the spatial variation of the one electronic states which appear in the polarization contribution in equation 20.

The separable form of the deformation potential in equation 9 allows us to write the self-energy term in equation 20 in the factorized form:

$$
\Pi\left(\lambda i, \lambda^{\prime} i^{\prime} ; \omega\right)=g_{\alpha m} \tilde{\Pi}\left(\alpha i, \beta i^{\prime} ; \omega\right) g_{\beta m^{\prime}}
$$

where $\alpha$ labels the phonon symmetry, and $\mathrm{n}$ is a phonon band index.

For the orientationally ordered phase we can drop the site index since we have only one molecule per unit cell. In addition since the icosahedral symmetry is still maintained, the reduced polarization term $\tilde{\Pi}$ is diagonal in the symmetry labels $\alpha$, so that

$$
\tilde{\Pi}(\alpha, \beta)=\delta_{\alpha \beta} \tilde{\Pi}(\alpha)
$$

Furthermore, since we have chosen the coupling matrices $(\Delta)$ to be irreducible under cubic symmetry we have the additional simplification, 


$$
\tilde{\Pi}(0) \neq \tilde{\Pi}(1)=\tilde{\Pi}(2)=\tilde{\Pi}(3) \neq \tilde{\Pi}(4)=\tilde{\Pi}(5)
$$

Ofcourse, the full $\Pi$ in equation 21 still has off-diagonal terms in the phonon band indices. Thus, in the orientationally ordered solid, the electron phonon interaction induces coupling only between phonons of the same symmetry in different bands.

However, in the orientationally disordered phases, off diagonal terms can and do appear even in $\tilde{\Pi}$ and they induce a significant mixing not only between phonons with different symmetries but also between phonons on different sites. In addition, there is also a significant variation in the the diagonal components of $\tilde{\Pi}$ from site to site as we see below.

We note that in equation 20 we have omitted the long wavelength contributions from the "intra-band" terms with $n=n^{\prime}$. In fact, for coupling to the (optical)Einstein modes relevant to our model, in which $\omega \rightarrow$ constant as $\mathrm{q} \rightarrow 0$, these contributions vanish. This is in striking contrast to the situation for coupling to acoustic modes, in which an additional intraband contribution also exists. Allen [31] has derived an approximate expression for $\operatorname{Im}(\Pi)$ for general wavevector $q$ which is of the form,

$$
\operatorname{Im}(\Pi(q))=\omega \sum_{k}|g(k, k+q)|^{2} \delta\left(E_{k}\right) \delta\left(E_{k+q}\right)
$$

The $q=0$ limit of this expression is indeed non-zero and results in a frequency $\operatorname{shift}\left(\Delta \omega_{n}\right)$ and $\operatorname{linewidth}\left(\gamma_{n}\right)$,

$$
\Delta \omega_{n}=N(0) \frac{g_{n}^{2}}{d_{n}} ; \gamma_{n}=\pi \omega_{n} N(0)^{2} \frac{g_{n}^{2}}{d_{n}}
$$

where $d_{n}$ is the degeneracy of the $n$th mode and $N(0)$ is the density of states at the fermi level. Although this result has been previously applied to the doped fullerides [0, 30], it is strictly applicable only in the limit that the Debye frequency for the relevant acoustic phonons is much smaller than the fermi energy, $E_{F}$, for the conduction charge. These conditions are violated for the fullerides where we have optical phonons with frequencies comparable to the fermi energy. In our treatment we will therefore consider only the self energy terms appearing in equation 22 . 
Once the self energy contributions are calculated using equation 20 the renormalized phonon Green's function is obtained by solving the Dyson equation

$$
D=D_{o}+D_{o} \Pi D
$$

For the orientationally ordered solid, the factorization in equation (21) allows us to reduce the matrix equation (26) to a scalar problem in each invariant subspace. For the disordered solid in which mixing between the various symmetry branches and sites occurs one has to solve the full matrix equation. However, the mixing between the $h_{g}$ and $a_{g}$ manifolds is expected to be small and we solve the equation in each subspace. Due to the factorisation in (21) the largest matrix which needs to be inverted for the $h_{g}$ phonons is only $5 \mathrm{M} \times 5 \mathrm{M}$ (where $\mathrm{M}$ is the number of sites).

For the orientationally ordered phase, the reduced polarization terms for the fully symmetric space $\alpha=0$ vanish identically. Thus a long wavelength excitation in the fully symmetric subspace provides a constant shift to the electronic potential, but cannot scatter electrons between conduction states. The remaining nonzero contributions in the irreducible $e_{g}$ and $t_{2 g}$ subspaces are displayed as functions of the phonon frequency in Figure 2. The imaginary part of the self energy (shown in the top panel) introduces a damping of the molecular modes, the real part (lower panel) describes the frequency shift due to the screening. We see that the overall shape of $\operatorname{Im} \tilde{\Pi}$ is similar in the two subspaces, peaking near $2000 \mathrm{~cm}^{-1}$. We find that the $t_{2 g}$ vibrations are coupled slighly more strongly, particularly at higher frequency. The most important consequence of this is that the real part of the self energy at low frequency ( below $1000 \mathrm{~cm}^{-1}$ ) is enhanced in the $t_{2 g}$ channel relative to the $e_{g}$ channel. This implies a "crystal field" splitting of the $\ell=2$ intramolecular modes with the $t_{2 g}$ frequency systematically suppressed relative to the $e_{g}$ frequency. Solving equation (25) we find that this splitting is of order $30 \mathrm{~cm}^{-1}$ for the second $h_{g}$ mode $\left(h_{g}(2)\right.$ in Table. 2) whose renormalised frequency is $372 \mathrm{~cm}^{-1}$. Finally, we note that the higher frequency Raman active modes overlap the region in which $\operatorname{Im} \tilde{\Pi}$ is large. This leads to a relatively large damping of the higher frequency Raman active vibrations. However, as we see below 
this situation is complicated somewhat by the orientational disorder of the host.

For the orientationally disordered phase the symmetry selection rules,(equations (22 and 23) no longer pertain and there are a large number of independent components of the reduced self-energy,గ̃. In Figure 3 we display a representative sample corresponding to the diagonal components of $\tilde{\Pi}$ corresponding to the same symmetry branch but different sites(i.e. $\tilde{\Pi}(i \alpha=5, i \beta=5)$ for various values of site $i)$. It can be seen that there is significant variation in $\tilde{\Pi}$ on different sites. This spread is expected to lead to an additional inhomogenous broadening of the phonon linewidths. This shape of these spectra can be understood simply. If we model the conduction band by a rectangular density of states and assume a contact interaction between the electrons and phonons which is independent of frequency and wavevector, the predicted $\operatorname{Im} \tilde{\Pi}(\omega)$ is obtained from a simple convolution of the filled and empty sectors of the one electronic spectrum. This yields a density fluctuation spectrum which is symmetric with a triangular shape, as indeed we find from our numerical computations.

The analogous self-energy terms in the $a_{g}$ subspace are shown in Figure 4 . The shape is again "triangular" and the overall scale is similar to that found for the $h_{g}$ subspace. Note however, that the self energy contributions are quite different owing to the variation of the amplitudes $g_{\alpha}$. It is nonetheless significant that these contributions are nonzero for the disordered phase. This reflects the fact that the long wavelength phonons in the folded Brillouin zone of the supercell are coupled to intracell charge fluctuations, and can thus be screened and damped by their coupling conduction electrons. The simplest example of this nonzero coupling in the symmetric subspace is the branch which is backfolded from $\mathrm{q}$ $=\left(\frac{2 \pi}{n a}, 0,0\right)$ in the extended zone, where $\mathrm{n}$ is the supercell period. This couples to the $\mathrm{x}-$ polarized density fluctuation of the conduction charge in the supercell.

We note that there are two intramolecular modes of $a_{g}$ symmetry: the breathing mode near $200 \mathrm{~cm}^{-1}$ and the pentagonal pinch near $1500 \mathrm{~cm}^{-1}$. These occur respectively well below, and near, the peak of $\operatorname{Im} \tilde{\Pi}(\omega)$ shown in Figure 4. Of course the phonon self energy depends on both the magnitude of $\tilde{\Pi}(\omega)$ and the coupling strength $g$. The coupling constants 
for the $a_{g}$ modes were computed in Ref. [⿴囗⿰丿㇄口 ( see Table 3) where it was found that the coupling to the breathing mode was very small while the coupling to the pentagonal pinch mode is quite substantial. The combination of this large coupling strength with our calculated $\tilde{\Pi}$ for the $a_{g}$ symmetry channel would predict a substantial broadening of the higher frequency $a_{g}$ mode. On the other hand experimentally $[7$ the increase in the linewidth of this phonon is small which seems to indicate that the actual coupling should also be small.

\section{INFRARED CONDUCTIVITY}

In this section we use the renormalized phonon Green's function developed in the previous section to calculate the frequency dependent conductivity of the coupled electron phonon system. We begin by considering the purely electronic contributions to the current current correlation function for the ordered and disordered phases. We then turn to the indirect contributions to the conductivity mediated by coupling through the dressed phonons.

\section{A. Electronic Contributions}

The real part of the frequency dependent conductivity is obtained within linear response theory as the imaginary part of the fourier transform of the current current correlation function. Since the wavelength of the probe is long on the scale of atomic dimensions it suffices to study the $q=0$ limit of these response functions. In our tight binding formalism, the paramagnetic term in the current operator is given by

$$
\vec{j}=i e \sum_{i j} \sum_{\mu \nu} \overrightarrow{\tau_{i j}} T_{\mu \nu}(i j) c_{i \mu}^{\dagger} c_{j \nu}
$$

where $\mathrm{T}$ is the intermolecular hopping matrix along the $\overrightarrow{\tau_{i j}}$-th bond. Thus the paramagnetic current-current correlation function is calculated from the Kubo formula

$$
\chi_{a b}(\omega)=i \int_{0}^{\infty} d t e^{-i \omega t}\left\langle\left[j_{a}(t), j_{b}(0)\right]\right\rangle
$$


where the angular brackets denote a thermodynamic average, and a, b are Cartesian indices. The frequency dependent conductivity, which is the linearized current in response to an external electric field is therefore

$$
\sigma_{a b}(\omega)=\frac{\operatorname{Im}\left(\chi_{a b}\right)}{\omega}
$$

For the noninteracting electronic Hamiltonian introduced in Section II, this correlation function can be readily calculated from the single particle eigenstates to yield

$$
\begin{aligned}
\left(\sigma_{e l}\right)_{a b}(\omega)= & \frac{1}{N} \sum_{k} \sum_{n n^{\prime}} \tilde{j}_{n n^{\prime}}^{a}(k) \tilde{j}_{n^{\prime} n}^{b}(k) \\
& \delta\left(E_{n k}-E_{n^{\prime} k}-\omega\right)\left(\frac{f\left(E_{n k}\right)-f\left(E_{n^{\prime} k}\right)}{E_{n k}-E_{n^{\prime} k}}\right)
\end{aligned}
$$

where $\mathrm{f}(\mathrm{E})$ is the Fermi-Dirac distribution, and $\tilde{j}_{n n^{\prime}}^{a}(k)$ is the matrix element of the a-th component of the current operator in (26) between single particle eigenstates $\mathrm{n}$ and $\mathrm{n}$ '. In this treatment which neglects the diamagnetic contributions to $\mathrm{j}$, the long wavelength low frequency Drude response needs to be evaluated with some care. One may proceed by studying the intraband contributions to $\mathrm{j}$ at finite wavevector $\mathrm{q}$, and extract the $\mathrm{q} \rightarrow 0$ limit:

$$
\left(\sigma_{a b}^{i n t r a}(\omega)\right)=\sum_{k n} \tilde{j}_{n n}^{a}(k) \tilde{j}_{n n}^{b}(k) \frac{\partial f_{k n}}{\partial E_{n k}} \delta(\omega)=D \delta(\omega)
$$

so that the weight of the Drude pole is obtained as a Fermi surface average

$$
D=\sum_{k n} \tilde{j}_{n n}^{a}(k) \tilde{j}_{n n}^{b}(k) \delta\left(E_{n k}\right)
$$

Rather than compute the Fermi surface average required in (30) our strategy will be to develop the sum rule for the total conductivity

$$
\left.\frac{2}{\pi} \int_{0}^{\infty} d \omega\left(\sigma_{e}\right)_{a b}(\omega)\right)=e^{2}\left\langle K_{a b}\right\rangle
$$

where, in the tight binding formalism $\left\langle K_{a b}\right\rangle$ is the ground state expectation value of the operator:

$$
K_{a b}=\left[R_{a}, j_{b}\right]=\sum_{i j} \sum_{\mu \nu}\left(\tau_{i j}\right)_{a}\left(\tau_{i j}\right)_{b} T_{\mu \nu}(i j) c_{i \mu}^{\dagger} c_{j \nu}
$$


Equation 34 is the generalized long wavelength f-sum rule [32] within our tight binding model. For the conducting phase, the interband paramagnetic part of the response function in 31 does not exhaust this sum rule, so that the residual weight provides the spectral weight in the Drude pole. For the free electron gas, the interband terms vanish identically, and then full spectral weight is then located in the pole at zero frequency. Our strategy is therefore to evaluate the sum rule, and the interband response function directly, and to extract the Drude piece by a comparision of their respective integrated spectral weights.

This method can be applied to both the orientationally ordered and disordered phases. The results for the frequency dependent conductivity are displayed in Figure 5, and the corresponding partitioning of the interband and "intraband" spectral weights are given in Table 4. For the orientationally ordered phase we observe a broad midinfrared continuum, extending from zero frequency to $4000 \mathrm{~cm}^{-1}$ peaking near $1600 \mathrm{~cm}^{-1}$. This peak can be roughly identified with a peak in the joint density of states do to the two primary peaks in the single particle density of states for the ordered phase. Approximately $40 \%$ of the total spectra weight is located in the Drude pole. We also remark that the interband response function extends to zero frequency, where it vanishes linearly with $\omega$. This is due to an interesting symmetry in the band structure for the orientationally ordered doped phase: the Fermi energy crosses a doubly degenerate band along the (111) directions in reciprocal space. This degeneracy is lifted as on shifts away from this symmetry direction, yielding a continuum of low frequency interband excitations which extends exactly to zero frequency. We remark that this degeneracy has some interesting consequences for the theory of superconductivity on such a Fermi surface which we have discussed in a previous paper 33.

Our results for a supercell containing quenched orientational disorder( also see [12 ) are shown in the lower panel in Figure 5. The supercell for this calculation contains 32 sites. Here we see that the interband peak is broadened and shifts to lower frequency where it overlaps the overdamped remnant of the Drude pole. The composite spectrum thus exhibits an overdamped midinfrared response function with a distinctly non-Drude lineshape. We 
remark that due to the artificial periodic boundary conditions in our supercell model, this spectrum retains a very small but nonzero spectral weight in a true Drude pole at zero frequency. This weight is nevertheless very small, and accounts for only $2 \%$ of the total spectral weight in this disordered model. This nonzero residual weight is nevertheless an artifact of the periodic boundary conditions, and will vanish as the size of the disordered cell is increased.

The results of Figure 5 demonstrate that merohedral disorder has a very strong effect on the dynamics of the conduction electrons, a point which has been emphasized in several previous studies [11 13]. The predicted low frequency low temperature conductivity is of order $3000 \mathrm{~S} / \mathrm{cm}$. This is rather small, but significantly larger than the experimental low temperature value of $1500 \mathrm{~S} / \mathrm{cm}$ [14. We believe that the discrepancy arises, at least in part, due to the effects of direct electron electron interactions which have been omitted from our treatment.

\section{B. Lattice Contributions}

Here we discuss corrections to the direct current current correlation function due to the coupling of the conduction charge to the intramolecular phonons. We are not concerned with the odd parity intramolecular vibrations which may be directly coupled to a long wavelength dipole active probe. Rather we will consider the even parity intramolecular modes which are indirectly coupled through the response conduction electrons. The appearance of these modes in the infrared is a very interesting phenomenon which, as we noted previously, can not occur for the orientationally ordered structure, nor indeed any for structure which preserves the inversion symmetry. Thus the appearance of these features in experimentally measured spectra is a fundamental signature of the microscopic breaking of inversion symmetry by orientational disorder. As we see below, these spectra are also quite sensitive to the details of the electron phonon coupling model, so that experimental measurements

of this effect might be used as a critical test of various microscopic models. The relevant 
microscopic process which indirectly couples the even parity intramolecular modes to light is illustrated diagramatically in Figure 6. As with the construction of the "direct" current current correlation function, the external field is directly coupled to density fluctuations of the conduction charge. These may in turn be coupled to the intramolecular modes through the vertex of Figure 1. The composite polarization term is one which thus correlates the current operator with the vibrational deformation potential and will be referred to as the "phonon charge." Thus the dressed phonon Green's function is indirectly coupled to the external field through this effective phonon charge, contributing to the full current current correlation function of the coupled electron phonon system. The microscopic theory of this process has been studied for low dimensional CDW systems [17,18] and for the conducting polymers [20]. In addition, it has been recently employed to explain the dopant induced infrared activity of the $t_{1 u}$ vibrations of the $C_{60}$ ions in the $A_{x} C_{60}$ compounds [34. As we noted above, the process represented in Figure 6 must vanish identically in an inversion symmetric structure. To explicity demonstrate this, it is conventient to examine the nonlocal conductivity for the coupled system in the position representation:

$$
\chi_{p h}(\omega)=\sum_{\lambda \lambda^{\prime}} V(\lambda i ; \omega+i \delta) D\left(\lambda i, \lambda^{\prime} j ; \omega+i \delta\right) V\left(\lambda^{\prime} j ; \omega-i \delta\right)
$$

where $\mathrm{D}$ is the renormalized phonon Green's function, $V(\lambda i)$ is the electronically induced phonon charge for the $\lambda$-th phonon on the i-th site. $V(\lambda i)$ is given by

$$
V(\lambda i ; \omega)=\sum_{i^{\prime}, i^{\prime \prime}} \int d E \operatorname{Tr}\left[j_{i^{\prime} i^{\prime \prime}} G_{i^{\prime \prime} i}(E) \Upsilon^{\lambda i} G_{i i^{\prime}}(E+\omega)\right]
$$

where $\mathrm{G}$ is a $3 \times 3$ matrix one particle Green's function in the space of orbital polarizations. In equation (35) the subscripts $\mathrm{i}$ are site indices and the trace is taken over the orbital indices. The operator $\Upsilon^{\lambda i}$ describes the intramolecular scattering of a conduction electron due to its linear coupling to the $\lambda$-th mode on site i:

$$
\Upsilon_{\mu \nu}^{\lambda i}=\Gamma^{\lambda} c_{\mu i}^{\dagger} c_{\nu i}
$$

Since the isolated fullerence molecule is inversion symmetric, it is clear that $\Upsilon^{\lambda i}$ is invariant under the inversion operation, $\mathrm{P}$. 


$$
P^{-1} \Upsilon^{\alpha i} P=\Upsilon^{\alpha i}
$$

Similarly, the current operator $\mathrm{j}$ appearing in equation should transform as a vector, which is odd under $\mathrm{P}$.

$$
P^{-1} \vec{j} P=-\vec{j}
$$

We note that on the lattice, the operator $\mathrm{j}$ involves the "off diagonal" elements of the electronic hopping operator $\mathrm{T}$ and thus equation (38) pertains only in the presence of inversion symmetry. Finally, for the inversion symmetric structure the one particle Green's function, and the two point density response function derived from it is even under parity. Thus the phonon charge, which involves a trace over a product of these three terms is odd under parity, and therefore vanishes in the $\mathrm{q} \rightarrow 0$ limit. For the disordered system, equation (36) is still true. However, the effective current operator in (26) is no longer odd under P, and furthermore, the two point density response function does not commute with P. In this case the effective phonon charge for an intramolecular "even parity" phonon is nonvanishing.

We now turn to an evaluation of the of indirect contribution to the conductivity given in equation (34). Our disordered system is modelled by a supercell, with periodic boundary conditions on the molecular orientations. In such a model

$$
\chi_{p h}(\omega)=\sum_{\lambda i, \lambda^{\prime} i^{\prime}} V(\lambda i ; \omega+i \delta) D\left(\lambda i, \lambda^{\prime} i^{\prime} ; \omega+i \delta\right) V\left(\lambda i^{\prime} ; \omega-i \delta\right)
$$

where now $i$ and $i^{\prime}$ are site indices, and the phonon charge is explicitly:

$$
\begin{aligned}
V(\lambda i ; \omega+i \delta)=\frac{1}{N} \sum_{k} \sum_{n n^{\prime}} \frac{\tilde{j}_{n n^{\prime}}(k) \tilde{\Gamma}_{n^{\prime} n}^{\lambda i}(k)}{E_{n k}-E_{n^{\prime} k}-\omega-i \delta} \\
\left(f\left(E_{n k}\right)-f\left(E_{n^{\prime} k}\right)\right)
\end{aligned}
$$

Note that in this model, crystal momentum is conserved modulo a reciprocal lattice vector of the supercell, and equations(40) and (41) therefore represent the appropriate $\mathrm{q}=0$ limits of these response functions.

The effective phonon charge in equation (41) is evaluated directly by numerical integration on a mesh of $12^{3}$ wavevectors in the folded Brillouin zone reciprocal to a supercell 
containing 32 molecular sites. The calculated contributions to Re $\sigma_{p h}$ due to coupling to the $h_{g}$ modes are displayed in Figure 7, where we have collected results for three different models for the electron phonon interaction. These are obtained for each of the coupling amplitudes $g_{\alpha}$ listed in Table 3. The contributions of equations (40) and (41) to the conductivity are additive and therefore each of these spectra should be superposed on the direct contribution to the conductivity shown in Figure 5(b). We see that for all the models, the coupling to the intramolecular "quadrupolar" modes produces a substantial modulation of the otherwise smooth featureless midinfrared conductivity. These modulations are correlated with the frequencies of the renormalized $h_{g}$ phonons, although it is found that the lineshapes are typically relatively broad and quite complex. The overall scale of these modulations is of order $100 \mathrm{~S} / \mathrm{cm}$ for all the models studied. Interestingly, we observe that the detailed structure in the modulated midinfrared conductivity is different for the various models. As we remarked earlier, the electron phonon models can be broadly grouped into two categories. In the first of these, the electron phonon coupling is dominated by the coupling to the two highest lying $h_{g}$ branches of the spectrum. The model due to Varma et. al. is a well known example from this group. These two highest lying modes are dominated by double bond stretching character on the fullerene, which are expected to couple strongly to the "antibonding" $t_{1 u}$ electronic state. In the second group of models, the coupling is distributed more uniformly among both the high frequency and lower frequency $h_{g}$ vibrations. The models derived by Schluter et. al. and by Faulhaber et. al. belong to this second category. These models require substantial coupling to the bond angle fluctuations and the conduction electrons. We find that in these latter models, but not in the former, the indirect contributions to the conductivtiy shows two well defined peaks in the range between 300 and $500 \mathrm{~cm}^{-1}$. In this region, the imaginary part of the phonon self energy is relatively small, and therefore the midfrequency intramolecular modes, if coupled to the conduction charge, show up as sharp features in the spectra. By contrast, for the former class of electron phonon models, with negligible coupling to the midfrequency modes, the sharp low frequency features are absent and the modulation spectra are "rugged" over a rather wide energy range, extending to the 
highest frequency modes near1700 cm-1.

The process illustrated in Figure 6, also leads to an indirect contribution to the conductivity from the high frequency, fully symmetric $a_{g}$ phonon branch. This resulting contribution to the conductivity is displayed in Figure 8 calculated using the theoretically calculated value from Ref. [4] Here the antisymmetric Fano type lineshape which is the signature of the resonance of this local mode with the pair continuum is clearly evident. The scale of this modulation is of order $100 \mathrm{~S} / \mathrm{cm}$, and its width is due to the relatively large coupling constant $g_{\alpha}$ for the second $a_{g}$ mode listed in Table 3. Experimentally, this mode is observed as a much narrower resonance, so that the microscopic prediction for the coupling strength for this mode appears to be overestimated.

Modulations of the midinfrared conductivity in $K_{3} C_{60}$ have recently been reported in experiments by Kuzmany et. al. [35,36] In Figure (9) we reproduce from this work, the real part of the full midinfrared conductivity, and the indirect phonon contribution, which is obtained by subtracting a smooth electronic background from the experimental measurements. We note that the experimental data in this Figure is taken at $\mathrm{T}=300 \mathrm{~K}$ and the scale of the conductivity over this range of frequency is known to depend on temperature. With this caveat, the modulations of $\operatorname{Re} \sigma$ due to coupling through the phonons is clearly evident in the experimental spectra. The two very sharp features near $600 \mathrm{~cm}$ and $1400 \mathrm{~cm}$ are odd parity modes, which are "directly" dipole active and thus not described by the indirect phonon charge model derived above. The remainder of the modulations in the conductivity are on a scale of typically $25-50 \mathrm{~S} / \mathrm{cm}$, and an additional sharp feature due to coupling to the high frequency symmetric $a_{g}$ mode is clearly evident. These modulations provide microscopic spectroscopic evidence for the local breakdown of inversion symmetry due to the orientational disorder in these samples. We also note that the experiment does not show any sharp phonon structure in the midfrequency range. Thus we believe that the type I electron phonon coupling models provide a more faithful representation of the experimental situation in these fullerides than the type II models. 


\section{SUMMARY}

In this paper we have focused on the interference between the intramolecular vibrational modes and the low lying single particle excitations measured in the midinfrared conductivity. Recent experimental data do provide some supporting evidence for this coupling in the doped

phases. However there are three aspects of the measured spectra which remain relatively poorly described by this theory, and to which we should call attention. First, the overall scale of the conductivity is incorrectly predicted in the theory. In fact the calculations overestimate the low frequency limit of $\operatorname{Re} \sigma(\omega)$ by nearly a factor of two. This is a serious difficulty in that the predicted value of $\sigma(\omega \rightarrow 0)$ is not rescaled by a simple rescaling of the conduction bandwidth. We believe that the failure to predict the correct scale of the measured conductivity is related to a deeper problem, and is likely associated with the neglect of direct electron electron interactions in the model. Second, careful experiments at very low frequency show a rather sharp minimum in the frequency dependent conductivity near $\omega \approx 100 \mathrm{~cm}^{-1}$. This is thought to separate a low frequency Drude like peak, from a rather broad midinfrared continuum. This sharp low frequency minimum is absent from the theory presented above, even in the presence of coupling to the phonons. It is possible that this originates in some strong couping to intermolecular vibrations neglected in our treatment. Finally, our discussion does not address the very interesting temperature dependence of the resistivity, which increases roughly linearly with temperature over a very broad temperature range, extending to $700 \mathrm{~K}$. At the highest temperatures studied, one may infer from these data that the effective mean free path is smaller than the radius of the $C_{60}$, a state of affairs which appears difficult to reconcile with the theoretical picture given above. Thus, while we believe that the phonon structure in the midinfared conductivity can be adequately explained by the theory presented here, there are a number of issues to be resolved in the experimental low frequency spectra. 


\section{ACKNOWLEDGMENTS}

This work was supported by the Department of Energy under Grant 91 ER 45118 and by the NSF under Grant 91 20668. HYC was supported by the Korean Science and Engineering Foundation (KOSEF) through the center for theoretical physics and through grant 931-0200003-2. 
Table 1 Hopping Amplitudes for the electronic Hamiltonian(Equation 1 obtained from Ref. [11]. These coefficients are multiplied by a scale factor, $t=0.014 \mathrm{meV}$ for doped fullerides.

\begin{tabular}{|c|c|c|c|c|c|c|c|}
\hline $\mathrm{A}$ & $\mathrm{B}$ & $\mathrm{C}$ & $\mathrm{D}$ & $\mathrm{E}$ & $\mathrm{F}$ & $\mathrm{X}$ & $\mathrm{Y}$ \\
\hline 0.01 & 0.38 & -2.29 & 2.09 & -2.36 & 0.38 & -0.63 & -0.49 \\
\hline
\end{tabular}

Table 2. Frequencies and line-widths for the bare phonons $\left(\right.$ in $\left.\mathrm{cm}^{-1}\right)$. The frequencies are as calculated in Ref. [28] and the linewidths are chosen to agree with experiment( [7]).

\begin{tabular}{|c|c|c|}
\hline mode & Frequency & Linewidth \\
\hline$h_{g}(1)$ & 244 & 4.2 \\
$h_{g}(2)$ & 412 & 5.5 \\
$h_{g}(3)$ & 560 & 7.5 \\
$h_{g}(4)$ & 750 & 9.0 \\
$h_{g}(5)$ & 1073 & 7.0 \\
$h_{g}(6)$ & 1202 & 7.6 \\
$h_{g}(7)$ & 1395 & 7.5 \\
$h_{g}(8)$ & 1508 & 9.5 \\
$a_{g}(1)$ & 507 & 2.5 \\
$a_{g}(2)$ & 1470 & 1.5 \\
\hline
\end{tabular}

Table 3. Coupling constants, $g_{\alpha n}$, as defined in Equation 11, for the $h_{g}$ and $a_{g}$ modes(in $\mathrm{meV})$ 


\begin{tabular}{|c|c|c|c|}
\hline mode & Ref. 3] & Ref. [4] & Ref. 28 \\
\hline$h_{g}(1)$ & 9.4 & 11.6 & 6.0 \\
\hline$h_{g}(2)$ & 7.1 & 13.5 & 21.1 \\
\hline$h_{g}(3)$ & 8.9 & 27.6 & 7.6 \\
\hline$h_{g}(4)$ & 0.0 & 20.5 & 28.7 \\
\hline$h_{g}(5)$ & 29.0 & 0.0 & 13.4 \\
\hline$h_{g}(6)$ & 0.0 & 0.0 & 18.0 \\
\hline$h_{g}(7)$ & 77.8 & 26.5 & 47.8 \\
\hline$h_{g}(8)$ & 46.0 & 44.0 & 37.8 \\
\hline$a_{g}(1)$ & - & 0.0 & - \\
\hline$a_{g}(2)$ & - & 52.0 & - \\
\hline
\end{tabular}

Table 4 Spectral weights in units of $t e^{2} / \hbar a$ (where $a$ is the lattice constant) corresponding to the interband and total electronic conductivities.

\begin{tabular}{|c|c|c|}
\hline Model & Interband weight & Total weight \\
\hline Ordered Crystal & 15.2 & 25.0 \\
Disordered Crystal & 26.6 & 27.1 \\
\hline
\end{tabular}




\section{REFERENCES}

[1] W. Kratschmer, L. D. Fostiroulus, and D. R. Huffman, Nature 347, 354(1990)

[2] O. Zhou, J. E. Fischer, N. Coustel, S. Kucia, Q. Zhu, A. R. M'Ghie, W. J. Romanov, J. P. M'Cauley, A. B. Smith III and D. E. Lox, Nature 351, 462(1991)

[3] C.M.Varma, J.Zaanen and K.Raghavachari, Science, 254, 989(1991)

[4] M.Schluter, M. Lanno, M. Needels and G. A. Baraff, Phys. Rev. Lett., 68, 526(1992)

[5] D. S. Bethune, G. Meijer, W. C. Tang, H. J. Rosen, W. G. Golden, H. Seki, C. A. Brown and M. S. de Vries, Chem. Phys. Lett. 179,181(1991)

[6] M. G. Mitch, S. J. Chase, J. S. Lannin, Phys. Rev. Lett. 68, 883(1992)

[7] P. Zhou, K. Wang, A. M. Rao, P. C. Ecklund, G. Dresselhaus, M. S. Dresselhaus, Phys. Rev. B 45, 10838(1992)

[8] P. W. Stephens, L. Mihaly, P. L. Lee, R. L. Whetten, S.-M, Huang, R. Kaner, F. Diedrich, K. Holczer, Nature(London) 351, 632(1991)

[9] M. Lanoo, G. A. Baraff, M. Schluter, Phys. Rev. B 44, 12106(1991)

[10] R.C. Haddon, L.E. Brus, and K.Raghavachari, Chem. Phys. Lett. 125, 459(1986); H. Weaver, J. L. Martins, T. Komeda, Y. Chen, T. R. Ohno, G. H. Kroll, and N. Troullier, Phys. Rev. Lett. 66, 1741(1991)

[11] M. P. Gelfand and J. P. Lu, Phys. Rev. Lett. 68, 1050(1992)

[12] M. P. Gelfand and J. P. Lu, Phys. Rev. B 46, 4397(1992)

[13] S. C. Erwin and E. J. Mele (to be published)

[14] L. Degiorgi, P. Wachter, G. Gruner, S. M. Huang, J. Wiley, and R. B. Kaner, Phys. Rev. B 46, 11250(1992)

[15] T. T. M. Palstra, R. C. Haddon, A. F. Hebard, and J. Zannen, Phys. Rev. Lett. 68, 
1054(1992); X.-D Xiang, J. G. Hou, G. Briceno, W. A. Vareka, R. Mostovoy, A. Zettl, V. H. Crespi, and M. L. Cohen, Science 256, 1190(1992)

[16] M. J. Rice, H-Y Choi, E. J. Mele, M. S. Deshpande (to appear in Phys. Rev. B)

[17] P. A. Lee, T. M. Rice, P. W. Anderson, Solid State Comm. 14, 703(1974)

[18] M. J. Rice, Phys. Rev. Lett. 37, 36(1976)

[19] M. J. Rice, L. Pietronero, P. Bruesch, Solid State Comm. 21, 757(1977)

[20] E. J. Mele and M. J. Rice, Phys. Rev. Lett. 45, 926(1980)

[21] T. Yildirim, S. Hong, A. B. Harris, E. J. Mele, Phys.Rev.B 48, 12262(1993)

[22] S. C. Erwin and W. E. Pickett, Science 254,842(1991)

[23] For a review, see W. E. Pickett, "Electrons and Phonons in $C_{60}$-based Materials", in Solid State Physics, Vol. 48, edited by H. Ehrenreich and F. Spaepen(Academic Press, New York, 1994), p. 225

[24] S. Satpathy, V. P. Antropov, O. K. Anderson, O. Jepsen, O. Gunnarsson and A. I. Liechtensten, Phys.Rev.B 46, 1773(1992)

[25] S. Satpathy, Chem. Phys. Lett. 130, 545(1986)

[26] G. Dresselhaus, M. S. Dresselhausr and D. C. Eklund ,Phys. Rev. B 45, 6923(1992)

[27] M. S. Deshpande and E. J. Mele(unpublished)

[28] J. C. R. Faulhaber, D. Y. K. Ko and P. R. Briddon, Phys. Rev. B 48,661(1993)

[29] R. A. Jishi and M. S. Dresselhaus, Phys. Rev. B

[30] M. Schluter, M. Lanoo, M. Needels, G. A. Baraff, and D. Tomanek, J. Phys. Chem. Solids 53, 1473(1992)

[31] P. B. Allen, Phys. Rev. B 6,2577 (1972) 
[32] P. F. Maldague, Phys. Rev. B 16, 2437(1977)

[33] S. C. Erwin and E. J. Mele, Phys. Rev. B 47, 2948(1993)

[34] H-Y Choi and M.J. Rice, Physical Review B 45, 10173 (1993)

[35] T. Pichler, M. Matus, H. Kuzmany, Solid State Comm. 86, 221(1993)

[36] T. Pichler, M. Matus, H. Kuzmany(preprint) 


\section{FIGURE CAPTIONS}

FIG.1. (a) The electron-phonon intereaction vertex in the Bloch orbital basis.(b) The one loop contribution to the phonon self energy,П. The electron and phonon propagators are represented by a solid and wavy line respectively.

FIG.2. Imaginary(upper panel) and Real(lower panel) parts of the reduced phonon selfenergy $(\Pi \tilde{(\alpha)})$ in the orientationally ordered solid corresponding to the $e_{g}(\alpha=1,2)$ and $t_{2 g}(\alpha=3,4,5)$ symmetry channels.

FIG.3. Imaginary(upper panel) and Real(lower panel) parts of the diagonal components of the reduced phonon self energy $(\tilde{\Pi}(i \alpha, i \alpha))$ for $h_{g}$ phonons in the disordered solid corresponding to $\alpha=5$ and a representative sample of sites $i$.

FIG.4. Imaginary(upper panel) and Real(lower panel) parts of the diagonal components of the reduced phonon self energy $(\tilde{\Pi}(i \alpha, i \alpha))$ for $a_{g}$ phonons in the disordered solid corresponding to $\alpha=0$ and a representative sample of sites $i$.

FIG.5. Real part of the pure electronic conductivity for the ordered(upper panel) and disordered(lower panel) solids

FIG.6. Diagrammatic representation of the process which generates the phonon contribution to the conductivity. The solid line denotes the electron propagator,the wavy line is the renormalised phonon propagator and the dotted line the photon propagator.

FIG.7. Results for the contribution of the $h_{g}$ modes to the conductivity using various models for the coupling constants $\left(g_{\alpha n}\right)$ listed in Table 3 .

FIG.8. Results for the contribution of the high frequency $a_{g}$ mode to the conductivity using the coupling calculated in Ref. [4] (see table 3).

FIG.9. (a)The experimental mid-infrared conductivity [35] with a fit representing the smooth electronic background (b) The contribution due to the phonons obtained by subtracting the electronic background. 